\title{
Genetic divensity and phylogenetic analysis of genus Paeonia based on nuclear ribosomal DNA ITS sequence
}

\author{
Yan-Lin Sun • Soon-Kwan Hong
}

Received: 19 August 2011 / Accepted: 5 September 2011

(c) Korean Society for Plant Biotechnology

\begin{abstract}
The genus Paeonia belongs to the family Paeoniaceae having significant medicinal and ornamental importance. The present investigation was undertaken with an aim to understand phylogenetic relationships of three Paeonia species (P. lactiflora, P. obovata, and P. suffruticosa) that are widely distributed in China, Korea, and Japan, using nuclear ribosomal DNA (nrDNA) internal transcribed spacer (ITS) sequence and to compare the phylogeny results with investigations reported earlier using existed sequences of the same species. The size variation obtained among sequenced nrDNA ITS region was narrow and ranged from 722 to $726 \mathrm{bp}$. The highest interspecific genetic distance (GD) was found between $P$. lactiflora and P. suffruticosa or P. obovata. The phylogram obtained using our nrDNA ITS sequences showed non-congruence with previous hypothesis of the phylogeny between section Paeonia and section Moutan of genus Paeonia. This result was supported by the phylogenetic relations showed in the phylogram constructed with existed sequences in NCBI. The present study suggested that $P$. obovata belonging to section Paeonia was phylogenetically closer to $P$. suffruticosa representing section Moutan of genus Paeonia than P. lactiflora belonging to section Paeonia. The main reason of the paraphyly of section Paeonia is thought to be nucleotide additivity directly caused by origin hybridization. This study provides more sequence sources of genus Paeonia, and will help for further studies in intraspecies population, and their phylogentic analysis and molecular evolution.
\end{abstract}

Keywords Genetic divergence, nrDNAITS region, Paeonia, phylogeny

Y.-L. Sun · S.-K. Hong $(\bowtie)$

Department of Bio-Health Technology, Kangwon National

University, Chuncheon, Kangwon, 200-701, Korea

e-mail: soonkwan@kangwon.ac.kr

S.-K. Hong

Institute of Bioscience and Biotechnology, Kangwon National

University, Chuncheon, Kangwon, 200-701, Korea

\section{Introduction}

Paeonia is the single genus in the family Paeoniaceae, which consists of ca. 35 species distributed mainly in warmtemperate regions of Europe and Asia (Wu et al. 2010). This genus is commonly divided into three sections: Moutan, Paeonia, and Onaepia (Pan 1979). The largest section, Paeonia, contains ca. 27 herbaceous species distributed in eastern and central Asia, the western Himalayas, and the European Mediterranean region, including $P$. lactiflore and P. obovate investigated in this study (Tank and Sang 2001). Section Moutan consists of five woody species, distributed in central and western China, including $P$. suffruticosa investigated in this study. The smallest section, Onaepia, consists of only two herbaceous species endemic to Pacific North America (Stern 1946; Pan 1979; Tank and Sang 2001). Many species of this genus have been used in traditional folk medicine, such as the roots of $P$. suffruticosa, $P$. lactiflora, $P$. obovata, and $P$. albiflora are the most important crude drugs in Traditional Chinese Medicine (TCM), and have been used as analgesic, sedative, and anti-inflammatory agents, and as remedies for cardiovarscular, extravasated blood, stagnated blood, and female genital diseases (Chang and But 1986; Wu 1990). Phytochemical investigations of Paeonia species have also revealed their main components exhibit significant biological and pharmacological activities (Tsai et al. 2008; Hu et al. 2010).

The World Health Organization has estimated that more than $80 \%$ of the world's population depends on herbal medicine for primary healthcare needs (Vines 2004). Medicinal plants have been playing more and more inherent and prominent roles in our life (Yuan et al. 2010). Due to long-term exploitation of wild medicinal herbs, many important Chinese traditional medicinal plants are becoming rare and endangered (Gao et al. 2005). In order to protect the medicinal plant resources and meet the increasing demand for plant-based drug and herbal remedies, the 
most popular medicinal plants have been cultivated under the supervision of Chinese government of grown spontaneously by farmers (Gao et al. 2005). However, before the farm cultivation of medicinal plants, their genetic diversity and phylogenetic analysis is required to make use safer and more correct.

Genus Paeonia has commonly been considered as a phylogenetically and taxonomically complex group (Stebbins 1938). In particular, section Paeonia, consisting of approximately 27 herbaceous species of both diploids and tetraploids, may have undergone complex reticulate revolution hat further obscured phylogenetic relationships (Sang et al. 1995, 1997a). The phylogeny of Paeonia was previously inferred based on nucleotide sequences from multiple genic and intergenic regions, including two loci of the low-copy nuclear gene alcohol dehydrogenase (Adh1 and Adh2), the nuclearencoded and chloroplast-expressed glycerol-3-phosphate acyltransferase (GPAT) gene, the cpDNA gene matK, two intergenic cpDNA spacers ( $t r n L-t r n F$ and $p s b A$-trnH), and the nrDNA ITS region (Sang et al. 1995; 1997a, b; Sang and Zhang 1999; Tank and Sang 2001). The molecular phylogenies support the monophyly of each of the three taxonomic sections of Paeonia. Despite the large amount of sequence data analyzed, some relationships remain unresolved, such as within subsection Vaginitae of section Moutan, and section Paeonia containing numerous hybrid species (Tank and Sang 2001). To further understand clearer molecular evolution and phylogenetic relationship of genus Paeonia, more accessions of more species, newer gene markers with higher rates of sequence divergence would be needed.

In this study, the genetic diversity of four accessions of three peony species (P. lactiflora, P. obovata, and P. suffruticosa) based on nrDNA ITS sequence variation was detected. Herbaceous peony, P. lactiflora (known as Radix Paeoniae Alba in medicine, and also called as baishao in Chinese; Hong and Pan 2001; Jiang et al. 2011; Ou et al. 2011), and woodland peony, P. obovata (called as caoshaoyao in Chinese; Hong et al. 2001), both belonging to the Paeonia section, are both widely distributed in China, Japan, Korea, and far east of Russia. Both species are used for representing the section Paeonia in this study. Tree peony, P. suffruticosa (known as Moutan Cortex in medicine, also called as mudanpi in Chinese;), belonging to the section Moutan, is widely overlapped distributions in China (Hong et al. 2010; He et al. 2010). This species is used for representing the section Moutan in this study. In the theory of TCM, P. lactiflora has a slightly cold nature, bitter and sour taste, and the root of this species is commonly used for medicinal purpose. It mainly distributes to treat the liver meridian and symptoms such as dizziness, costal part pain, stomach ache, limb spasm and ache, blood deficiency with dim yellow skin, irregular menses and spontaneous perspiration (National Committee of Pharmacopoeia 2005). In addition, herbaceous peony has large and beautiful flowers, so the flower of this plant is mainly cultivated for ornamental purpose in China and has been introduced to Japan, America, and Europe. The flower of $P$. suffruticosa is known as the king of flowers in China (Hong et al. 1998), and its root cortex has been widely used as an herbal medicine in Asia for a long time, to treat such diseases as atherosclerosis, infection, and inflammation as well as cutaneous disease (Jiang et al. 2007; Hong et al. 2010). Little information about the use of $P$. obovata is found, except of being used as a painkiller by the Ainu people (Batchelor and Miyabe 1893).

According to the phylogenetic analysis using nrDNA ITS sequence, it was suggested that $P$. obovata belonging to section Paeonia of genus Paeonia was phylogenetically closer to $P$. suffruticosa representing section Moutan, but not the species, $P$. lactiflora that belongs to the same section Paeonia as P. obovata. This results proved the taxonomically complexity of section Paeonia, and might provide more sequence sources to study the taxonomically complex section Paeonia and section Moutan.

\section{Materials and methods}

Plant materials

A total of four voucher specimens, consisting of three Paeonia species [P. lactiflora (2 accessions), and P. obovata, both belonging to section Paeonia, and $P$. suffruticosa belonging to section Moutan] were included in this study. Leaves were picked from four vouch specimens, and deposited in Department of Bio-Health Technology, Plant Developmental and Engineering Lab (PDEL), Kangwon National University, Korea, for molecular study. The names of these specimens are listed here together with the collectors' number have been deposited in the National Centre for Biotechnology Information (NCBI) under the GenBank accession numbers of JN572147 JN572150. The detailed information of the plant materials investigated in this study was shown in Table 1. 
Table 1 Taxa, voucher specimen information, origin, and GenBank accession numbers for the investigated plant materials

\begin{tabular}{cccc}
\hline Taxon & Voucher specimen & Origin and collection number & ITS GenBank accession number \\
\hline Paeonia lactiflora Pall. & CHA & Korea, cult. and Hong & JN572147 \\
Paeonia obovata Maxim. & SHAN & Korea, cult. and Hong (2) & JN572148 \\
Paeonia suffruticosa Andrews & MO & Korea, cult. and Hong (3) & JN572149 \\
Paeonia lactiflora Pall. & CHA1 & Korea, cult. and Hong (1) & JN572150 \\
\hline
\end{tabular}

Isolation of DNA, amplification and sequencing

DNA extractions were performed by using the modified cetyltrimethylammonium bromide (CTAB) method described by Doyle and Doyle (1987). Universal ITS primer sets ITS5, 5'-GAA AGT AAA AGT CGT AAC AAG G-3' and ITS4, 5'-TCC TCC GCT TAT TGA TAT GC-3' were used to amplify the nrDNA ITS region including ITS1, 5.8S rRNA, ITS2 regions (White et al. 1990). PCR amplification was conducted using this set of primers with the following program: 35 cycles of denaturation at $95^{\circ} \mathrm{C}$ for $1 \mathrm{~min}$, annealing at $53^{\circ} \mathrm{C}$ for $1 \mathrm{~min}$, and a final extension step at $72^{\circ} \mathrm{C}$ for $1.5 \mathrm{~min}$. All PCR products were purified before DNA sequence analysis using a QIAquick PCR Purification Kit (QIAGEN, Korea, Cat. No. 28104) according to the manufacturer's instructions. Purified PCR products were then sequenced at MACROGENE Advancing through Genomics (Korea, http://dna.macrogen.com/kor/).

Sequence editing and alignment

For editing and assembly of the complementary strands, the software program DNAMAN version 6.0 (Lynnon Biosoft Corporation, USA, www.lynon.com) was used. Analogue of our sequences and nucleotide sequence comparisons were detected with Basic Local Alignment Search Tool (BLAST) network services against databases (http://www.ncbi.nlm.nih. gov/). The multiple sequence alignment of ITS region (ITS1, 5.8S rRNA gene and ITS2) of all the four Paeonia species was performed also using DNAMAN version 6.0 software, to detect single nucleotide polymorphisms.

Phylogenetic analysis

The phylogenetic relationships among the four Paeonia species was estimated after the construction of a phylogram based on multiple sequence alignment of ribosomal DNA ITS sequence with the DNAMAN version 6.0 software (Lynnon Biosoft Corporation, USA, www.lynon.com). The relevant region lengths (ITS1, 5.8S rRNA gene and ITS2) of each sequence were calculated, and the $\mathrm{G}+\mathrm{C}$ contents of each sequence were calculated with the help of BIOEDIT software. Genetic distance (GD) was obtained with the help of MEGA software and mean GD of the intraspecific distance was calculated by sum of individual GD divide by number of samples.

\section{Results and discussion}

The nrDNA ITS region has been used in numerous systematic studies at genus and species levels of a wide array of plant taxa (Wen and Zimmer 1996; Alice and Campbell 1999; Sun et al. 2010a, b). The two internal spacers, ITS1 and ITS2 are located between the 18S nrRNA gene and the 26S nrRNA gene, and are referred as nrDNA ITS region combined with 5.8S nrRNA gene located between ITS1 and ITS2 (Baldwin 1992). Generally, ITS1 and ITS2 are less than 300 by in length, and the 5.S subunit is almost invariant in length within angiosperms around $160 \mathrm{bp}$, making the entire ITS region less than $700 \mathrm{bp}$ (Sudheer Pamidimarri et al. 2009). Its small size, ease of amplification and rapid concerted evolution made nrDNA ITS region as a useful tool for deducing phylogetic relation. In this study, nrDNA ITS sequence were amplified with primers ITS4 and ITS5 from P. lactiflora, P. obovata, and P. suffruticosa showed very narrow size variations and ranged from 722 to 726 bp (data now shown). The PCR products consisted of partial 18S rRNA gene sequence, ITS1, 5.8S rRNA gene, and ITS2 complete sequence, and partial 26S rRNA gene sequence (GenBank accession number, JN 572147 JN572150 in NCBI). Among these four accessions of peony species, there was single base-pair sequence variation only, observed in the ITS2 region between $P$. suffruticosa and other species (Table 2). In the full ITS sequence, there was only one nucleotide variation existing in the ITS1 region between CHA and CHA1 (Fig. 1), but not in the 5.8S rRNA gene and ITS2 region sequence (Figs. 2, 3; Table 2). Whereas, in interspecific level, $22 \mathrm{bp}$ and $19 \mathrm{bp}$ of nucleotide variations were obtained in the full ITS region sequence with $P$. obovata and $P$. suffruticosa, respectively (Figs. 1-3; Table 2). These nucleotide variations consisted of $4 \mathrm{bp}$ and $5 \mathrm{bp}$ specific-nucleotide site differences of $P$. obovata and $P$. suffruticosa in the ITS1 region, respectively 
Table 2 Sequence length, nucleotide variation, and G + C content (\%) of ITS1, 5.8S rRNA gene, and ITS2 region

\begin{tabular}{|c|c|c|c|c|c|c|c|c|c|c|c|c|c|c|c|c|}
\hline \multirow{2}{*}{$\begin{array}{c}\text { Vouch } \\
\text { specimen }\end{array}$} & \multicolumn{3}{|c|}{ Sequence length (bp) } & \multicolumn{3}{|c|}{ Nucleotide variation (bp) } & \multicolumn{3}{|c|}{ Variation percentage $(\%)$} & \multicolumn{3}{|c|}{ Varied nucleotide percentage $(\%)$} & \multicolumn{4}{|c|}{$\mathrm{G}+\mathrm{C}$ content $(\%)$} \\
\hline & ITS1 & $\begin{array}{c}5.8 \mathrm{~S} \\
\text { rRNA }\end{array}$ & ITS2 & ITS1 & $\begin{array}{c}5.8 \mathrm{~S} \\
\text { rRNA }\end{array}$ & ITS2 & ITS1 & $\begin{array}{c}5.8 \mathrm{~S} \\
\text { rRNA }\end{array}$ & ITS2 & ITS1 & $\begin{array}{c}5.8 \mathrm{~S} \\
\text { rRNA }\end{array}$ & ITS2 & ITS1 & $\begin{array}{c}5.8 \mathrm{~S} \\
\text { rRNA }\end{array}$ & ITS2 & $\begin{array}{c}\text { Total ITS } \\
\text { region }\end{array}$ \\
\hline CHA & 265 & 159 & 228 & 0 & 0 & 0 & \multirow{4}{*}{5.28} & \multirow{4}{*}{0.63} & \multirow{4}{*}{$\begin{array}{c}5.70 \text { to } \\
5.73\end{array}$} & 0 & 0 & 0 & 55.47 & 53.46 & 57.02 & 55.52 \\
\hline SHAN & 265 & 159 & 228 & 10 & 1 & 11 & & & & 71.43 & 100 & 84.62 & 56.98 & 52.83 & 58.77 & 56.60 \\
\hline MO & 265 & 159 & 227 & 11 & 0 & 8 & & & & 78.57 & 0.00 & 61.54 & 56.60 & 53.46 & 59.91 & 56.99 \\
\hline CHA1 & 265 & 159 & 228 & 1 & 0 & 0 & & & & 7.14 & 0.00 & 0.00 & 55.85 & 53.46 & 57.02 & 55.67 \\
\hline
\end{tabular}

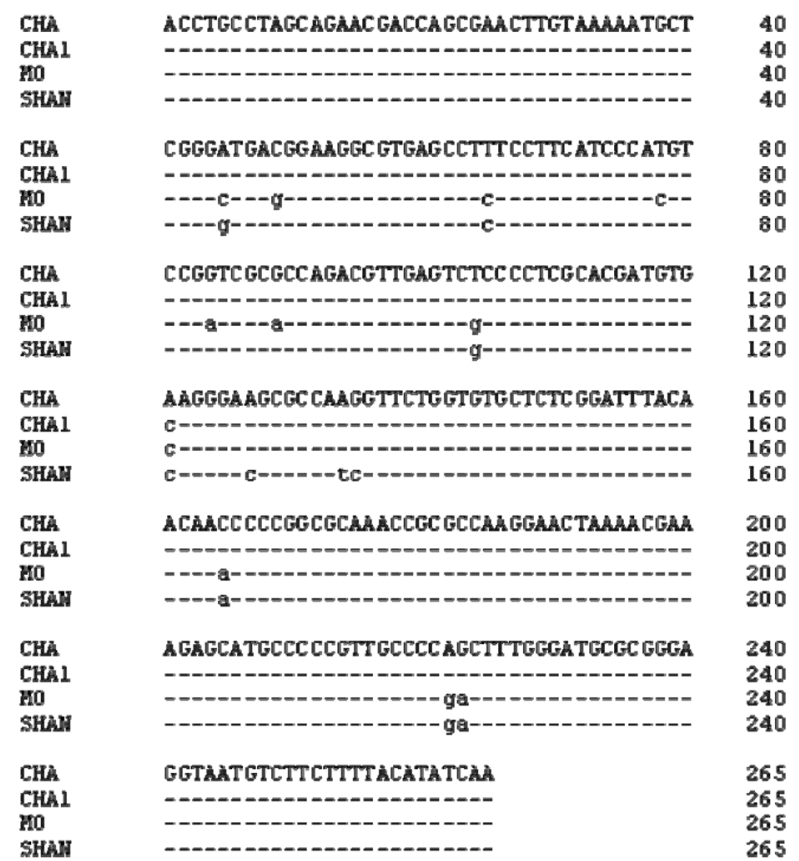

Fig. 1 DNA alignment of ITS1 region sequence of four accessions of three peony species. - means identity with the first line sequence

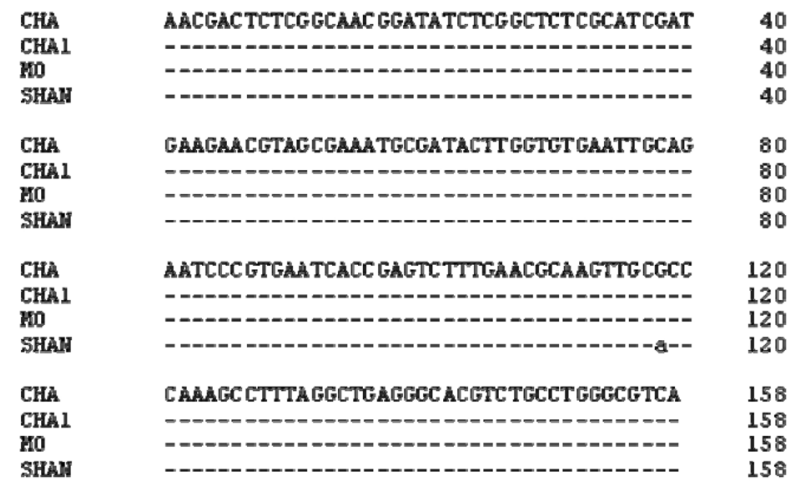

Fig. 2 DNA alignment of 5.8S rRNA gene sequence of four accessions of three peony species. - means identity with the first line sequence

(Fig. 1), only 1 bp specific-difference observed in the $5.8 \mathrm{~S}$ rRNA gene of $P$. obovata (Fig. 2), and 2 bp and $6 \mathrm{bp}$ specific-differences in the ITS2 region of $P$. obovata and P. suffruticosa, respectively (Fig. 3). Intraspecific $\mathrm{G}+\mathrm{C}$

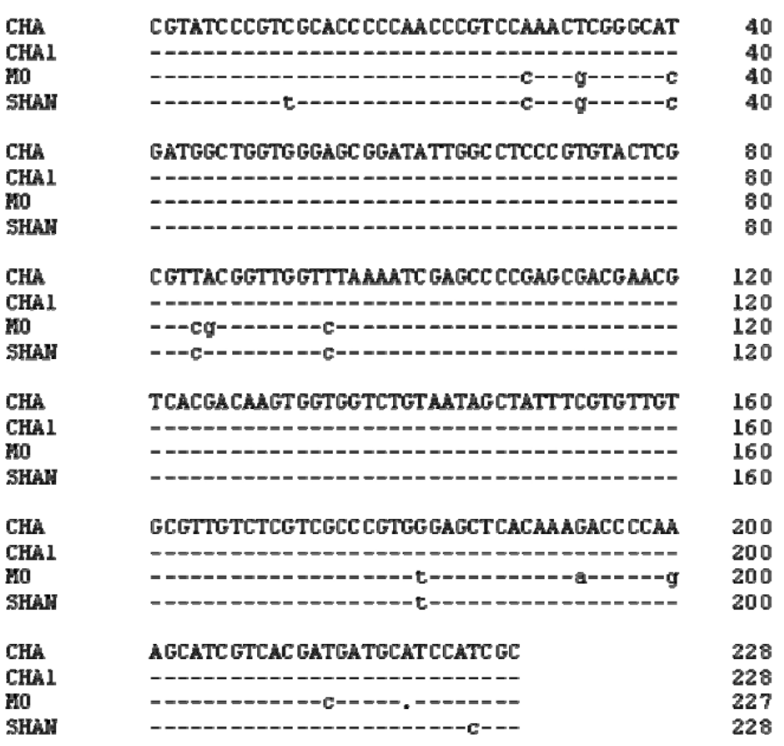

Fig. 3 DNA alignment of ITS2 region sequence of four accessions of three peony species. - means identity with the first line sequence

content of the sequences showed very narrow variations, and only found in the ITS1 region caused by the nucleotide differences (Table 2). Contrarily, the interspecific $\mathrm{G}+\mathrm{C}$ content showed to be varied remarkable: the minimum interspecies $\mathrm{G}+\mathrm{C}$ content of the ITS1 region appeared in P. lactiflora and the maximum in P. obovata; the $\mathrm{G}+\mathrm{C}$ content of $P$. obovata in the 5.8S rRNA gene was specifically different from that of other species; the minimum interspecies $\mathrm{G}+\mathrm{C}$ content of the ITS2 region also appeared in P. lactiflora and the maximum in $P$. suffruticosa (Table 2). The total ITS region $\mathrm{G}+\mathrm{C}$ content ranged from 55.52 (P. lactiflora) to $56.99 \%$ ( $P$. suffruticosa).

The intraspecific GD was found to be 0.008 between two accessions of P. lactiflora (Table 3). The highest interspecific GD (0.046) was found between P. lactiflora and $P$. suffruticosa or $P$. obovata, while the least interspecific GD (0.038) was found between $P$. suffruticosa and $P$. obovata.

The phylogram was generated based on our nrDNA ITS sequences from four accessions of three species, suggesting that $P$. obovata (SHAN) was phylogenetically closer to $P$. 
Table 3 Pair wise inter and intraspecific genetic distance (GD) of genus Paeonia based on nrDNAIT Ssequence

\begin{tabular}{ccccc}
\hline & CHA & CHA1 & MO & SHAN \\
\hline CHA & 0.000 & & & \\
CHA1 & 0.008 & 0.000 & & \\
MO & 0.046 & 0.045 & 0.000 & \\
SHAN & 0.042 & 0.046 & 0.038 & 0.000 \\
\hline
\end{tabular}

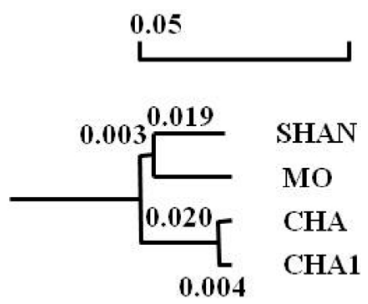

Fig. 4 Phylogenetic tree of genus Paeonia generated using our nrDNA ITS sequences according to the one-parameter model by Jukes and Cantor (1969). Number means relevant branch length

suffruticosa (MO) than P. lactiflora (CHA and CHA1; Fig. 4). However, it is commonly known that $P$. lactiflora and $P$. obovata belong to section Paeonia of genus Paeonia, while $P$. suffruticosa belongs to section Moutan of the same genus, Paeonia. Previous phylogenetic hypotheses strongly supported the monophyly of three sections, Paeonia, Onaepia, and Moutan (Sang et al. 1995, 1997a, b). Sang et al. (1997b) combined the AdhlA, ITS, and matK DNA sequence data to produce a phylogenetic tree that is congruent with the monophyly of section Paeonia, Onaepia, and Moutan. According to the phylogeny analysis of genus Paeonia using GPAT gene sequence, Tank and Sang (2001) disproved the monophyly of section Paeonia and section Onaepia that subsection Foliolatae of section Paeonia is phylogenetically closer to $P$. californica, representing section Onaepia than subsection Paeonia of section Paeonia. Despite there were some controversy about the monophyly of section Paeonia and section Onaepia, the monophyly of section Paeonia and section Moutan were not doubted. However, in this study, the monophyletic group formed by $P$. obovate representing section Paeonia and $P$. suffruticosa representing section Moutan was parallel with another monophyletic group formed only by species of section Paeonia, that was not congruent with previous phylogenetic relations of both section Paeonia and section Moutan.

To well understand the phylogenetic relationship of $P$. lactiflora, $P$. suffruticosa, and $P$. obovata, the phylogram was generated based on our ITS region sequences and existed ITS sequences of these species in NCBI (Fig. 5).

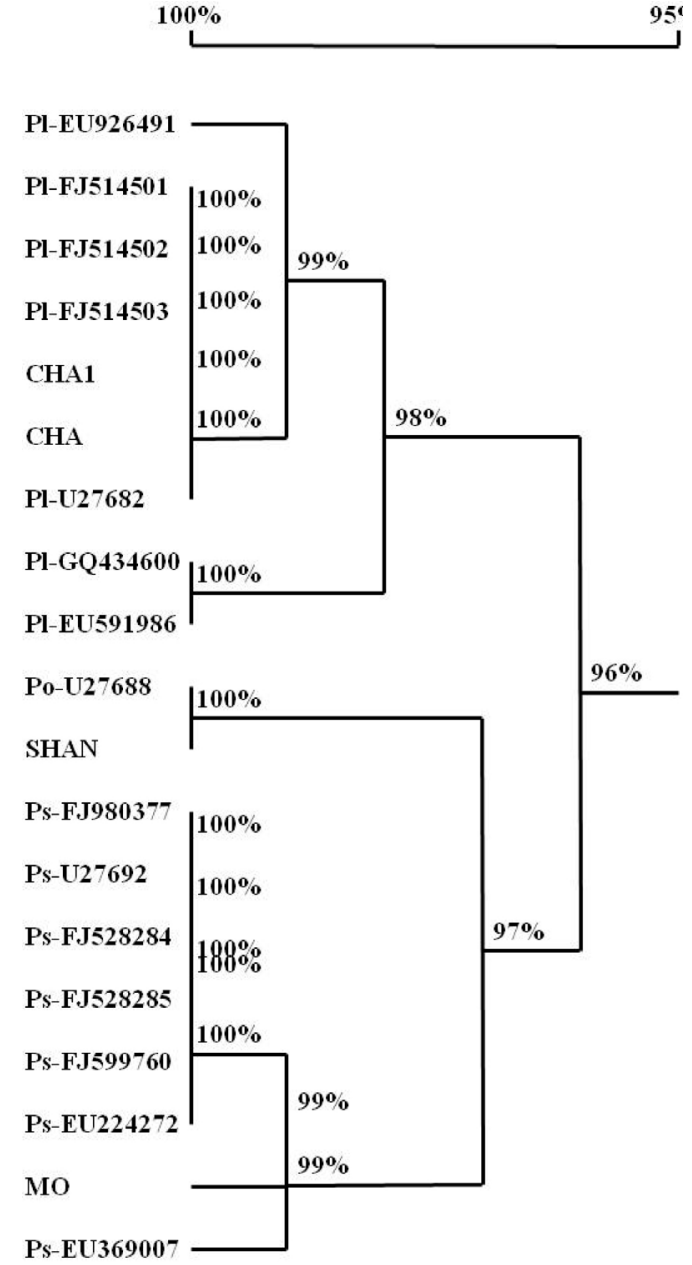

Fig. 5 Phylogenetic tree of genus Paeonia generated using nrDNA ITS sequence. Pl means existed ITS sequence from $P$. lactiflora in NCBI GenBank data; Po means existed ITS sequence from $P$. obovata in NCBI GenBank data; and Ps means existed ITS sequence from $P$. suffruticosa in NCBI GenBank data

And this phylogram obtained the same result as the phylogram of our sequence did (Fig. 4). There were seven existed ITS sequence from $\mathrm{P}$. lactiflora, seven from $P$. suffruticosa, and only one from $P$. obovata in all. Our $P$. lactiflora ITS sequences (CHA and CHA1) showed very high identity with some existed P. lactiflora sequences including FJ514501, FJ514502, FJ514503, and U27682, and formed a major clade with other three sequences of the same species (EU926491, GQ434600, and EU591986), with $98 \%$ of similarity (Fig. 4). Our P. obovata ITS sequence (SHAN) were very identical to the exclusive sequence of this species existed in NCBI, U27688. $P$. suffruticosa sequences formed a monophyletic group containing our sequence (MO), and the least similarity with each other was found to be $99 \%$. The $P$. suffruticosa group formed another major clade with the $P$. obovata monophyletic group, with the least similarity of $97 \%$. Although $P$. suff- 
ruticosa belongs to section Moutan of genus Paeonia, different from P. lactiflora and P. obovata belonging to section Paeonia of genus Paeonia, P. obovata was phylogenetically closer to $P$. suffruticosa than $P$. lactiflora based on nrDNA ITS sequence.

Our results were not congruent with previous phylogeny of P. obovata and section Moutan by Sang et al. (2004). In the report of Sang et al. (2004), several individuals of $P$. obovata were used to investigate the phylogenetic relations of section Paeonia and section Moutan represented by $P$. suffruticosa, $P$. decomposita, $P$. rockii, and $P$. delavayi, and found to be monophyletic especially in phylogeny tree generated using the $A d h 2$ gene sequence. As known, Paeonia section Paeonia has long been considered to be a taxonomically difficult group, presumably because of reticulate evolution and hybridization directly resulting in nucleotide additivity (Stebbins 1948; Sang et al. 1995). Thus, few ambiguous nucleotide sites were found in the sequences obtained directly from a PCR pool, and no nucleotide substitutions were detected among populations of a species (Sang et al. 1995), that directly magnified roles of some nucleotide variations to induce great difference in phylogram based on DNA sequences. In addition, hybridization caused the molecular diversification among populations of $P$. anomala, indicated that $P$. anomala is a homoploid hybrid that originated from a across between $P$. veitchii and P. lactiflora (Pan et al. 2007). Sequence polymorphism was found at the $A d h 1$ and $A d h 2$ loci within populations of $P$. anomala, which were grouped with $P$. veitchii and $P$. lactiflora, respectively. And $P$. anomala was grouped with $P$. veitchii on the ITS and GPAT phylogenies but with $P$. lactiflora on the chloroplast phylogeny. Thus increased sampling of populations and more molecular markers were required to investigate in studies of phylogenetic analysis.

The present study proves the usefulness of the nrDNA ITS sequence in phylogenetic analysis of genus Paeonia and pave way for future phylogentic and/or evolutionary studies among the groups belongs to the family Paeoniaceae. The sequence data generated will help for further studies in intraspecies population, and their phylogentic origins, biogeographical and molecular evolutionary studies. However, the further understanding of the phylogeny of genus Paeonia needs to construct and compare more DNA sequence sources in the future. As the standard of human life and the demands of medicinal herbs are increasing, this study also would help the use accuracy and safety of this species.

\section{Acknowledgement}

This work was supported by Nutraceutical Bio Brain Korea 21 Project Group.

\section{Literature cited}

Alice LA, Campbell CH (1999) Phylogeny of Rubus (Rosaceae) based on nuclear ribosomal DNA internal transcribed spacer region sequences. Am J Bot 86:81-97

Baldwin BG (1992) Phylogenetic utility of the internal transcribed spacers of nuclear ribosomal DNA in plants: an example from the compositae. Mol Phyl Evol 1:3-16

Batchelor J, Miyabe K (1893) Ainu economic plants. Transactions of the Asiatic Society of Japan (R. Meiklejohn \& Co.) 51:198240

Chang HM, But PH (1986) Pharmacology and Applications of Chinese Meteria Medica. World Scientific Press, Singapore, vol. 1, p. 165

Doyle JJ, Doyle JL (1987) A rapid DNA isolation procedure for small quantities of fresh leaf tissue. Phytochem Bull 19:11-15

Gao W, Jia W, Gao X, Wang R, Xiao P (2005) In vitro culture and cultivation of Chinese medicinal plants for industrial utilization and genetic resource conservation. Plant Genet Resur 3:116126

He CN, Peng Y, Xu LJ, Liu ZA, Gu J, Zhong AG, Xiao PG (2010) Three new oligostilbenes from the seeds of Paeonia suffruticosa. Chem Pharm Bull 58:843-847

Hong DY, Pan KY (2001) Paeoniaceae. In: Wu ZY, Raven PH (eds) Flora of China. Science Press and Missouri Botanic Garden Press, Beijing, China, vol. 6, pp 127-132

Hong DY, Pan KY, Rao GY (2001) Cytogeography and taxonomy of the Paeonia obovata polyploidy complex (Paeoniaceae). Plant Systemat Evol 227:123-136

Hong DY, Pan KY, Yu H(1998) Yingpingmudan, the wild relative of the king of flower, Paeonia suffruticosa Andrews. Acta Phytotax Sin 36:515-520

Hong MH, Kim JH, Na SH, Bae HS, Shin YC, Kim SH, Ko SG (2010) Inhibitory effects of Paeonia suffruticosa on allergic reactions by inhibiting the NF-kappaB/IkappaB-alpha signaling pathway and phosphorylation of ERK in an animal model and human mast cells. Biosci Biotechnol Biochem 74:1152-1156

Hu SL, Shen G, Zhao WG, Wang F, Jiang XD, Huang DB (2010) Paeonol, the main active principles of Paeonia moutan, ameliorates alcoholic steatohepatitis in mice. J Ethnopharmacol 128:100-106

Jiang DX, Chen YS, Hou XT, Xu JF, Mu X, Chen W (2011) Influence of Paeonia lactiflora roots extract on cAMP-phosphodiesterase activity and related anti-inflammatory action. J Ethnopharmacol 137:914-920

Jiang S, Nakano Y, Yatsuzuka R, Ono R, Kamei C (2007) Inhibitory effects of Moutan cortex on immediate allergic reactions. Biol Pharm Bull 30:1707-1710 
Jukes TH, Cantor CR (1969) Evolution of protein molecules. In: Munro HN (ed) Mammalian Protein Metabolism. Academic Press, New York, pp 21-132

National Committee of Pharmacopoeia (2005) Pharmacopoeia of the Peoples's Republic of China (Part one). Chemical Industry Press, Beijing, p 69

Ou TT, Wu CH, Hsu JD, Chau CC, Lee HJ, Wang CJ (2011) Paeonia lactiflora Pall inhibits bladder cancer growth involving phosphorylation of Chk2 in vitro and in vivo. J Ethnopharmacol 135: 162-172

Pan J, Zhang D, Sang T (2007) Molecular phylogenetic evidence for the origin of a diploid hybrid of Paeonia (Paeoniaceae). Am J Bot 94:400-408

Pan KY (1979) Paeonia. In: Flora Reipublicae Siniccae. Science Press, Beijing, vol. 27, pp 37-59

Sang T, Crawford DJ, Stuessy TF (1995) Documentation of reticulate evolution in peonies (Paeonia) using internal transcribed spacer sequences of nuclear ribosomal DNA: implications for biogeography and concerted evolution. Proc. Natl. Acad. Sci. USA 92:6813-6817

Sang T, Crawford DJ, Stuessy TF (1997a) Chloroplast DNA phylogeny, reticulate evolution, and biogeography of Paeonia (Paeoniaceae). Am J Bot 84:1120-1136

Sang T, Donoghue MJ, Zhang DM (1997b) Evolution of alcohol dehydrogenase genes in peonies (Paeonia): phylogenetic relationships of putative nonhybrid species. Mol Biol Evol 14:994-1007

Sang T, Pan J, Zhang DM, Ferguson D, Wang C, Pan KY, Hong DY (2004) Origins of polyploids: an example from peonies (Paeonia) and a model for angiosperms. Biol J Linn Soc 82: 561-571

Sang T, Zhang DM(1999) Reconstructing hybrid speciation using sequences of low copy nuclear genes: hybrid origins of five Paeonia species based on Adh gene phylogenies. Systematic Botany 24:148-163

Stebbins GL Jr. (1948) Review of 'A study of the genus Paeonia by F. C. Stern.'. Madroño 9:193-199

Stern FC (1946) A study of the genus Paeonia. The Royal Horticultural Society, London
Sudheer Pamidimarri DVN, Chattopadhyay B, Reddy MP (2009) Genetic divergence and phylogenetic analysis of genus Jatropha based on nuclear ribosomal DNA ITS sequence. Mol Biol Rep 36:1929-1935

Sun YL, Park WG, Kwon OW, Hong SK (2010a) The internal transcribed spacer rDNA specific markers for identification of Zanthoxylum piperitum. Afr J Biotechnol 9:6027-6039

Sun YL, Park WG, Kwon OW, Hong SK (2010b) Ribosomal DNA internal transcribed spacer 1 and internal transcribed spacer 2 regions as targets for molecular identification of medically important Zanthoxylum schinifolium. Afr J Biotechnol 9:4661-4673

Tank DC, Sang T (2001) Phylogenetic utility of the glycerol-3phosphate acyltransferase gene: evolution and implications in Paeonia (Paeoniaceae). Mol Phylogenet Evol 19:421-429

Tsai HY, Lin HY, Fong YC, Wu JB, Chen YF, Tsuzuki M, Tang CH (2008) Paeonol inhibits RANKL-induced osteoclastogenesis by inhibiting ERK, p38 and NF-kappaB pathway. Eur J Pharmacol 588:124-133

Vines G(2004) Herbal Harvests with a Future: Towards Sustainable Sources for Medicinal Plants. Report for Plantlife International Salisbury, Wiltshire: The Wild Plant Conservation Charity

Wen J, Zimmer EA (1996) Phylogeny and biogeography of Panax L. (Ginseng genus, Araliaceae): inferences from ITS sequences of nuclear ribosomal DNA. Mol Phylogenet Evol 6:167-177

White TJ, Bruns T, Lee S, Taylor J (1990) Amplification and direct sequencing of fungal ribosomal RNA genes for phylogenetics. In: Innis MA, Gelfand DH, Sninsky JJ, White TJ (eds) PCR protocols-a guide to methods and applications. Academic Press, San Diego, Calif, pp 315-322

Wu CY (1990) Outline of New China Herbals. Shanghai Science and Technology Press, Shanghai, China, p. 207

Wu SH, Wu DG, Chen YW (2010) Chemical constituents and bioactivities of plants from the genus Paeonia. Chem Biodivers 7: 90-104

Yuan QJ, Zhan ZY, Hu J, Guo LP, Shao AJ, Huang LQ (2010) Impacts of recent cultivation on genetic diversity pattern of a medicinal plant, Scutellaria baicalensis (Lamiaceae). BMC Genetics 11:29-43 